\title{
Variations with modest effects have an important role in the genetic background of type 2 diabetes and diabetes-related traits
}

This article has been corrected since Advance Online Publication, and a corrigendum is also printed in this issue.

\author{
Hayato Fujita ${ }^{1}$, Kazuo Hara ${ }^{1}$, Nobuhiro Shojima ${ }^{1}$, Momoko Horikoshi ${ }^{1}$, Minoru Iwata ${ }^{2}$, Yushi Hirota ${ }^{3}$, \\ Kazuyuki Tobe ${ }^{2}$, Susumu Seino ${ }^{3}$ and Takashi Kadowaki ${ }^{1}$
}

The aim of the present study was to explore the role of variations with modest effects (previously identified by a large-scale meta-analysis in European populations) in the genetic background of type 2 diabetes (T2D) and diabetes-related traits in a Japanese population. We enrolled 2632 Japanese subjects with T2D and 2050 non-diabetic subjects. We analyzed nine single-nucleotide polymorphisms (SNPs), including rs340874 (PROX1), rs4607517 (GCK), rs2191349 (DGKB-TMEM195), rs7034200 (GLIS3), rs10885122 (ADRA2A), rs174550 (FADS1), rs11605924 (CRY2), rs10830963 (MTNR1B) and rs35767 (IGF1). rs340874 (PROX1) and rs174550 (FADS1) were significantly associated with T2D $(P=0.0078,0 R: 1.12$; and $P=0.0071$, OR: 1.12, respectively). Subjects with more risk alleles related to nine SNPs had an increased risk of T2D $(P=0.0017)$, as well as a higher fasting plasma glucose level $(P=0.018)$, higher $\mathrm{HbA}_{1 \mathrm{c}}$ level $(P=0.013)$ and lower HOMA- $\beta(P=0.033)$ compared with subjects who had fewer risk alleles. We identified a significant association of a SNP of FADS1 and a SNP near PROX1 with T2D in a Japanese population. The present findings suggest that inclusion of SNPs with a tendency to increase the disease risk captured more of the genetic background of T2D than that revealed by only assessing significant SNPs. Journal of Human Genetics (2012) 57, 776-779; doi:10.1038/jhg.2012.110; published online 20 September 2012

Keywords: common disease; single-nucleotide polymorphism; type 2 diabetes

\section{INTRODUCTION}

Type 2 diabetes (T2D) is a complex disorder characterized by hyperglycemia that results from impaired pancreatic $\beta$-cell function and decreased action of insulin on its target tissues. T2D is one of the most common polygenic disorders, in which each genetic variation has a partial and additive effect. ${ }^{1}$ Over 50 susceptibility loci for T2D have been identified through genome-wide association studies (GWAS), ${ }^{2-4}$ most of which were conducted in European populations. $K C N Q 1, U B E 2 E 2$ and $C 2 C D 4 A / B$ are among the few T2D loci discovered in non-European populations. ${ }^{5-7}$

Recently, new genetic loci were found to be associated with fasting glucose homeostasis in a meta-analysis of 21 GWAS of European nondiabetic subjects (the Meta-Analyses of Glucose and Insulin-related traits Consortium: MAGIC). ${ }^{4}$ The contribution of these new loci should be investigated further, because it is well known that there are significant differences in the frequencies of some genetic variations among different ethnic groups. Possibly because of the modest size of the effect of most variations, however, it is hard to replicate the results of studies that have analyzed large samples in other populations. In fact, only a handful of loci (CDKAL1, CDKN2A/B, IGF2BP2, TCF7L2, SLC30A8, HHEX, KCNJ11 and IRS-1) have been confirmed to be associated with T2D in Japanese populations. ${ }^{6,8-10}$

In the meantime, arguments against the common disease-common variant hypothesis have been proposed, suggesting that rare variants with a large effect have a substantial role in the genetic architecture of common diseases. So far, we only have limited examples of rare variants conferring a markedly increased risk of common diseases. It has also recently been proposed that a very large number of common variants with effects that cannot be detected by studies employing a conventional sample size may be involved in the genetic architecture of common diseases, which is the so-called 'infinitesimal' hypothesis. ${ }^{11}$

Even if individual small effects are not detectable by a single SNP (single-nucleotide polymorphism) analysis, we may be able to detect their cumulative effect by combined SNP analysis.

Accordingly, the aim of the present study was to determine whether the loci identified in the MAGIC study comprise part of

${ }^{1}$ Department of Diabetes and Metabolic Diseases, Graduate School of Medicine, The University of Tokyo, Tokyo, Japan; ${ }^{2}$ First Department of Internal Medicine, Toyama University, Toyama, Japan and ${ }^{3}$ Department of Internal Medicine, Division of Diabetes, Metabolism and Endocrinology, Kobe University Graduate School of Medicine, Kobe, Japan

Correspondence: Dr K Hara, Department of Diabetes and Metabolic Diseases, Graduate School of Medicine, University of Tokyo, 7-3-1 Hongo Bunkyo-ku, Tokyo 113-0033, Japan.

E-mail: thara-tky@umin.ac.jp

Received 11 April 2012; revised 10 July 2012; accepted 8 August 2012; published online 20 September 2012 
the genetic component of diabetes-related traits and T2D in a Japanese population by using combined SNP analysis.

\section{MATERIALS AND METHODS}

\section{Cases and controls}

Written informed consent was obtained from all the participants. We enrolled 1200 subjects with T2D aged $65.5 \pm 9.5$ years (body mass index (BMI): $24.3 \pm 3.7 \mathrm{~kg} \mathrm{~m}^{-2}$ (mean \pm s.d.)) who attended the University of Tokyo Hospital (Tokyo, Japan). The control group consisted of 855 persons aged $69.5 \pm 6.8$ years (BMI: $24.5 \pm 3.2 \mathrm{~kg} \mathrm{~m}^{-2}$ ) who participated in an annual health check conducted by the Hiroshima Atomic Bomb Casualty Council Health Management Center (Hiroshima, Japan). We also enrolled 722 subjects with T2D aged $64.9 \pm 11.1$ years (BMI: $24.6 \pm 3.9 \mathrm{~kg} \mathrm{~m}^{-2}$ ) and 763 controls aged $66.7 \pm 10.6$ years (BMI: $22.7 \pm 3.3 \mathrm{~kg} \mathrm{~m}^{-2}$ ) who attended the University of Toyama Hospital (Toyama, Japan) and 710 patients aged $60.9 \pm 10.2$ years (BMI: $24.1 \pm 3.7 \mathrm{~kg} \mathrm{~m}^{-2}$ ) and 432 controls aged $75.2 \pm 8.0$ years (BMI: $21.5 \pm 3.6 \mathrm{~kg} \mathrm{~m}^{-2}$ ) from the University of Kobe (Kobe, Japan).

Diabetes was diagnosed according to the World Health Organization criteria. ${ }^{12}$ Exclusion criteria included positivity for antibody to glutamic acid decarboxylase or diabetes secondary to (i) liver dysfunction, (ii) steroids or other drugs that might elevate glucose levels, (iii) malignancy or (iv) any monogenic disorder known to cause diabetes. Inclusion criteria for the control group were as follows: (i) $\mathrm{HbA}_{1 \mathrm{c}}<6.2 \%$, (ii) fasting plasma glucose $<7.0 \mathrm{mmoll}^{-1}$ and (iii) postprandial plasma glucose $<11.1 \mathrm{mmoll}^{-1}$. $\mathrm{HbA}_{\mathrm{lc}}$ was estimated according to the method of the Japan Diabetes Society and converted to the corresponding National Glycohemoglobin Standardization Program (NGSP) value.

\section{Genotyping}

We genotyped SNPs that showed a significant association with diabetes-related traits or phenotypes in the MAGIC study. SNPs with a minor allele frequency of less than 0.05 in the Japanese population (HapMap JPT) were excluded from this study. We also excluded SNPs for which the association with T2D has already been reported in samples from Tokyo or Toyama. ${ }^{7,13-15}$ As a result, a total of nine SNPs (rs340874, rs4607517, rs2191349, rs7034200, rs10885122, rs174550, rs11605924, rs10830963 and rs35767) were genotyped.

SNP genotyping was performed by using the TaqMan assay (Applied Biosystems, Foster City, CA, USA). The success rates of the assay was $>95 \%$.

\section{Statistical analysis}

In the non-diabetic subjects, the genotype distributions of all SNPs were in Hardy-Weinberg equilibrium $(P \geqslant 0.05)$. Using the non-diabetic subjects, we performed a quantitative trait analysis to assess the association between each SNP and indexes of glucose homeostasis (fasting plasma glucose, fasting insulin, $\mathrm{HbA}_{1 c}, \mathrm{HOMA}-\beta$ and HOMA-IR). Differences of the genotype distribution between the diabetic and non-diabetic groups were also assessed by logistic regression analysis with an additive model. We performed the above analyses separately on groups from the University of Tokyo, University of Toyama and University of Kobe, and then performed joint analyses.

We analyzed the combined effect of multiple SNPs. Risk alleles were selected from the MAGIC study and we calculated how many risk alleles there were in each sample for the nine SNPs analyzed in this study. Samples with 12 or more risk alleles were classified as the high-risk group, samples with 9-11 risk alleles were classified as the medium-risk group and samples with 8 or fewer risk alleles were classified as the low-risk group. Differences in the distribution of the cases and controls between the risk groups were analyzed. Using the control samples, we analyzed differences of diabetes-related traits between the risk groups. After excluding two SNPs (PROX1 and FADS1) that were significantly associated with T2D in the present study, we also analyzed the combined influence of the remaining seven SNPs. In this analysis, the highrisk group was defined as having 10 or more risk alleles, the medium-risk group had 7-9 risk alleles and the low-risk group had 6 or fewer risk alleles. We assessed the differences in the prevalence of T2D by using a logistic regression model and diabetes-related traits by using a linear regression model. We calculated odds ratios per one risk-group increase for T2D and effect sizes per 1 risk-group increase for diabetes-related traits. Effect sizes and s.e. were calculated for groups from the University of Tokyo, University of Toyama and University of Kobe, after which combined analysis was performed.

As dependent variables in the linear regression models, we used logtransformed data for fasting insulin, HOMA-IR and HOMA- $\beta$, whereas untransformed data were used for fasting plasma glucose and $\mathrm{HbA}_{1 \mathrm{c}}$.

Multiple corrections were done by the Benjamini-Hochberg method. ${ }^{16}$ A false discovery rate (FDR) of 0.05 was employed as the significance threshold.

These analyses were performed with R software (http://www.R-project.org).

\section{RESULTS}

We found that 2 SNPs (rs340874 and rs174550) were significantly associated with T2D (Table 1). In the case of rs340874 (PROX1), a minor allele was associated with an increased risk of T2D $(P=0.0078$, FDR-adjusted $P=0.035$, OR: $1.12,95 \%$ CI: $1.03-1.22)$. For rs 174550 (FADS1), a major allele was associated with an increased risk of T2D ( $P=0.0071$, FDR-adjusted $P=0.035$, OR: $1.12,95 \%$ CI: $1.03-1.22$ ). In addition, rs2191349 (DGKB-TMEM195) was nominally associated with T2D ( $P=0.035$, FDR-adjusted $P=0.11$, OR: 1.10 , 95\% CI: $1.01-$ 1.21). No associations were observed between T2D and the remaining loci in our study population.

In the non-diabetic subjects, there was a significant association with $\mathrm{HbA}_{1 \mathrm{c}}$ for rs4607517 (near GCK, $P=0.00056$, FDR-adjusted $P=0.0050$ ) (Table 2 ). However, none of the loci were significantly associated with fasting plasma glucose, fasting plasma insulin, HOMA-IR or HOMA- $\beta$ (Supplementary Tables 1-4).

The higher risk groups had an increased risk of T2D $(P=0.0017$, OR: $1.17,95 \%$ CI: $1.06-1.29$ ) in the combined analysis (Table 3). It is noteworthy that the higher risk groups had an increased risk of T2D even after excluding the two SNPs (near PROX1 and at FADS1) that were significantly associated with T2D in the individual SNP analyses ( $P=0.011$, OR: $1.14,95 \%$ CI: 1.03-1.27) (Table 3). The higher risk groups had higher fasting plasma glucose levels and $\mathrm{HbA}_{1 \mathrm{c}}$ levels ( $P=0.018$ and $P=0.013$, respectively) (Table 4$)$. The higher risk groups had a lower HOMA- $\beta(P=0.033)$, but no differences of HOMA-IR or fasting plasma insulin levels were found between the risk groups.

\section{DISCUSSION}

In the present study, we analyzed nine SNPs that were previously shown to be associated with diabetes-related traits by the MAGIC study. We found that a SNP of FADS1 and a SNP near PROX1 were significantly associated with an increased risk of T2D in a Japanese population. We also identified an association between a SNP of GCK and elevation of $\mathrm{HbA}_{1 \mathrm{c}}$ in a non-diabetic Japanese population.

To our knowledge, this is the first study to identify the association of T2D with a SNP at FADS1. In the MAGIC study, FADS1 was associated with fasting plasma glucose levels within the physiological range, but not with pathological glucose levels. ${ }^{4}$ However, this was not the case in the present study because FADS1 was associated with susceptibility to T2D in our Japanese population.

The present study is the first to identify a SNP near PROX1 related to susceptibility to T2D in a Japanese population. The contribution of a SNP near PROX1 to the pathogenesis of T2D has now been demonstrated across different ethnicities because the risk allele in the present study was directionally consistent with those found in other populations. ${ }^{4,17}$

In the combined SNP analysis, the higher risk groups had an increased risk of T2D. This suggests that these SNPs had an additive effect on the risk of T2D. Unexpectedly, an increased risk of T2D was 
Table 1 Association of the SNPs with type 2 diabetes

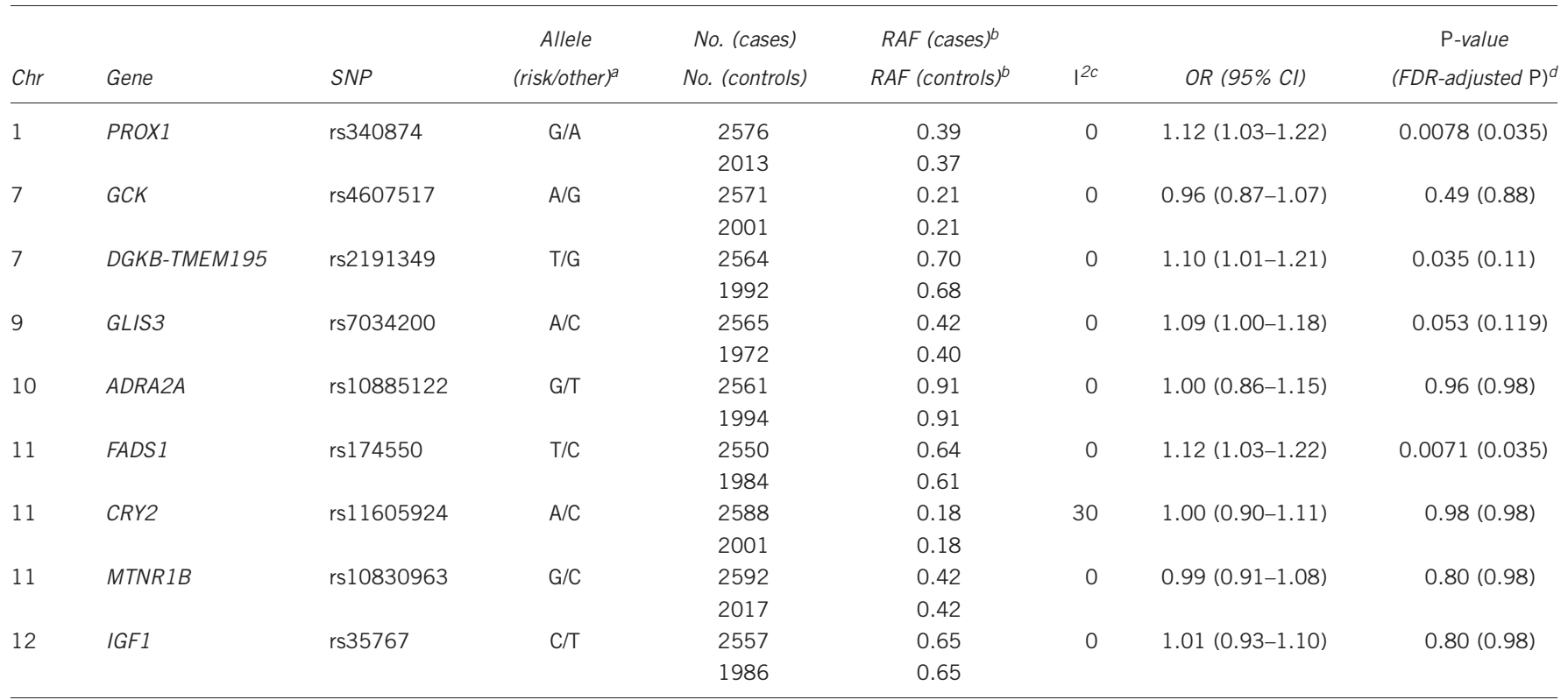

Abbreviations: $\mathrm{Cl}$, confidence interval; FDR, false discovery rate; OR, odds ratio; SNP, single-nucleotide polymorphism.

aThe risk alleles were determined according to the results in European populations. ${ }^{4}$

${ }^{\mathrm{b}} \mathrm{RAF}$ denotes the risk allele frequency.

${ }^{c}$ Heterogeneity was assessed by using the $R$ index.

dFDR-adjusted $P$-value for multiple hypotheses, using the Benjamini-Hochberg method.

Table 2 Association of the SNPs with $\mathrm{HbA}_{1 \mathrm{c}}$

\begin{tabular}{|c|c|c|c|c|c|c|}
\hline Chr & Gene & SNP & $\begin{array}{l}\text { Alleles } \\
\text { (effect/ } \\
\text { other) }^{a}\end{array}$ & No. & $\begin{array}{c}\text { Effect (\%) } \\
(\text { s.e. })^{b}\end{array}$ & $\begin{array}{l}\mathrm{P} \text {-value }(F D R \text { - } \\
\text { adjusted } \mathrm{P})^{c}\end{array}$ \\
\hline 1 & PROX1 & rs340874 & $\mathrm{G} / \mathrm{A}$ & 1837 & $0.013(0.017)$ & $0.450(0.59)$ \\
\hline 7 & GCK & rs4607517 & $A / G$ & 1830 & $0.040(0.012)$ & $0.00056(0.0050)$ \\
\hline 7 & $\begin{array}{l}\text { DGKB- } \\
\text { TMEM195 }\end{array}$ & rs2191349 & $T / G$ & 1822 & $0.001(0.015)$ & $0.94(0.94)$ \\
\hline 9 & GLIS3 & rs7034200 & $A / C$ & 1816 & $0.008(0.015)$ & $0.060(0.23)$ \\
\hline 10 & $A D R A 2 A$ & rs10885122 & $\mathrm{G} / \mathrm{T}$ & 1825 & $0.027(0.016)$ & $0.10(0.23)$ \\
\hline 11 & FADS1 & rs 174550 & $\mathrm{~T} / \mathrm{C}$ & 1824 & $0.015(0.010)$ & $0.13(0.23)$ \\
\hline 11 & CRY2 & rs11605924 & $\mathrm{A} / \mathrm{C}$ & 1843 & $0.009(0.012)$ & $0.47(0.59)$ \\
\hline 11 & MTNR1B & rs10830963 & $\mathrm{G} / \mathrm{C}$ & 1851 & $0.011(0.017)$ & $0.52(0.59)$ \\
\hline 12 & $\mid G F 1$ & rs35767 & $\mathrm{C} / \mathrm{T}$ & 1831 & $0.016(0.010)$ & $0.11(0.23)$ \\
\hline
\end{tabular}

Abbreviations: FDR, false discovery rate; SNP, single-nucleotide polymorphism.

aThe effect allele was determined according to the results in European populations. ${ }^{4}$

${ }^{\mathrm{b} P e r-a l l e l e ~ e f f e c t ~ a n d ~ s . e . ~ f o r ~ q u a n t i t a t i v e ~ t r a i t s ~ w e r e ~ e s t i m a t e d . ~}$

cFDR-adjusted $P$-value for multiple hypotheses, using the Benjamini-Hochberg method.

also found even after we excluded the two SNPs that were significantly associated with T2D. Subjects with multiple risk alleles in the MAGIC study had a higher fasting plasma glucose, higher $\mathrm{HbA}_{1 \mathrm{c}}$ and lower HOMA- $\beta$, even though few or none of their SNPs were significantly associated with diabetes-related traits in the individual analyses. These results suggest that SNPs with an effect that is too small to detect in individual SNP analyses have a role in the genetic architecture of T2D and support the infinitesimal hypothesis. It seems that we are able to detect their cumulative effect by combined SNP analysis.

In this study, correction for multiple testing was done by the Benjamini-Hochberg method. This controls the FDR and is less conservative than methods that control the family-wise error rate (FWER),
Table 3 Type 2 diabetes in the risk groups

\begin{tabular}{|c|c|c|c|c|c|c|}
\hline$S N P S^{a}$ & $\begin{array}{l}\text { No. }(\text { low } \\
\text { risk) }\end{array}$ & $\begin{array}{l}\text { No. } \text { (medium }^{\text {risk) }} \\
\text { c }\end{array}$ & & $1^{2 e}$ & OR $(95 \% C I)^{f}$ & P-value \\
\hline 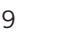 & 701 & 2241 & 1007 & 0 & 1.17 (1.06-1.29) & 0.0017 \\
\hline 7 & 588 & 2584 & 900 & 0 & $1.14(1.03-1.27)$ & 0.011 \\
\hline \multicolumn{7}{|c|}{$\begin{array}{l}\text { Abbreviations: Cl, confidence interval; OR, odds ratio; SNP, single-nucleotide polymorphism. } \\
\text { aNumber of SNPs used to calculate the genetic risk. Nine SNPs were used to calculate the } \\
\text { genetic risk (above). Seven SNPs were used after removing two SNPs that were significantly } \\
\text { associated with type } 2 \text { diabetes in the individual SNP analyses (below). } \\
\text { bThe low-risk group had } 8 \text { or fewer risk alleles around the nine SNPs (above), or } 6 \text { or fewer risk } \\
\text { alleles around the seven SNPs (below). } \\
\text { 'The medium-risk group had } 9-11 \text { risk alleles around the nine SNPs (above), or 7-9 risk } \\
\text { alleles around the seven SNPs (below). } \\
\text { dThe high-risk group had } 12 \text { or more risk alleles around the nine SNPs (above), or } 10 \text { or more } \\
\text { risk alleles around the seven SNPs (below). } \\
\text { eHeterogeneity was assessed by using the } R \text { index. } \\
\text { fOdds ratios per one risk-group increase were calculated. }\end{array}$} \\
\hline
\end{tabular}

such as Bonferroni's correction. The seven SNPs with no significant association in the individual analyses had a significant influence on T2D in the combined analysis, which suggests that methods controlling the FWER may be too conservative and methods controlling the FDR may be preferable in such studies. Similarly, the standard GWAS threshold of $5 \times 10^{-8}$ may be too strict. It could be useful to calculate a genetic risk score by using all SNPs beyond the nominal statistical thresholds in GWAS and determine whether the genetic score predicts the phenotype in another sample, but further investigation of this point is needed.

The higher risk groups had lower HOMA- $\beta$, but there were no differences of HOMA-IR or fasting plasma insulin levels between the 
Table 4 Diabetes-related traits in the risk groups.

\begin{tabular}{|c|c|c|c|c|c|c|}
\hline Phenotype & $\begin{array}{l}\text { No. (low } \\
\text { risk) }\end{array}$ & $\begin{array}{l}\text { No. } \text { (medium }^{\text {risk) }} \\
\text { r }\end{array}$ & $\begin{array}{c}\text { No. (high } \\
\text { risk)c }\end{array}$ & $1^{2 d}$ & Effect (s.e.) ${ }^{e}$ & $\begin{array}{c}\mathrm{P}- \\
\text { value }\end{array}$ \\
\hline $\begin{array}{l}\text { FPG } \\
\left(\mathrm{mmol} \mathrm{I}^{-1}\right)^{f}\end{array}$ & 226 & 685 & 288 & 0 & $0.054(0.023)$ & 0.018 \\
\hline $\mathrm{HbA}_{1 \mathrm{c}}(\%)$ & 275 & 837 & 350 & 0 & $0.026(0.011)$ & 0.013 \\
\hline $\begin{array}{l}\text { Fasting insulin } \\
\left(\mathrm{pmoll}^{-1}\right)\end{array}$ & 227 & 687 & 286 & 0 & $-0.022(0.023)$ & 0.34 \\
\hline HOMA-IR & 219 & 658 & 279 & 0 & $-0.010(0.024)$ & 0.67 \\
\hline HOMA- $\beta$ & 219 & 657 & 279 & 0 & $-0.051(0.024)$ & 0.033 \\
\hline
\end{tabular}

The low-risk group had 8 or fewer risk alleles around the nine SNPs.

bThe medium-risk group had 9-11 risk alleles around the nine SNPs.

dHeterogeneity was assessed by using the $R$ index.

EEffect sizes per one risk-group increase were calculated.

fFasting plasma glucose.

risk groups. This was consistent with the results of the MAGIC study, which showed that most of the nine SNPs were associated with HOMA- $\beta$ and only two SNPs were associated with HOMA-IR or fasting insulin.

We could not replicate all of the MAGIC study findings. One possible reason is that the linkage disequilibrium (LD) relationships of disease-susceptibility alleles may differ between populations. Because the LD block in the CRY2 regions is much larger in CEU than in JPT according to the HapMap database, it is possible that the causative SNPs under investigation are in different LD blocks in the Japanese population but are in the same LD in an European population. The fact that Japanese subjects with T2D tend to be leaner and less hyperinsulinemic than Europeans may be one of the reasons why SNPs associated with fasting insulin levels or HOMA-IR were not replicated.

Because our sample size did not have enough power to detect susceptibility genes with modest effect sizes, it cannot be denied that the other loci investigated in the current study could have some role in predisposing Japanese to T2D. In the quantitative analysis of the association between each SNP and diabetes-related traits except for $\mathrm{HbA}_{10}$, only 1344 samples were included, which is one of the reasons we could not replicate the associations between SNPs and diabetesrelated traits, except for that between $G C K$ and $\mathrm{HbA}_{1 \mathrm{c}}$.

In conclusion, the present study was the first to demonstrate that a SNP of FADS1 contributes to susceptibility to T2D. We also identified a significant association between T2D and a SNP near PROX1. Furthermore, our results indicated that analysis of SNPs with a small contribution to disease risk could capture more of the genetic background of T2D than assessment of only the significant SNPs.

\section{CONFLICT OF INTEREST}

The authors declare no conflict of interest.

\section{ACKNOWLEDGEMENTS}

We thank the participating doctors and staff from collaborating institutions for providing DNA samples. We also thank N Miyama and M Yamaguchi for technical assistance. Furthermore, we thank the technical staff at the Laboratory of the First Department of Internal Medicine, Faculty of Medicine, Toyama University; and Division of Diabetes, Metabolism and Endocrinology, Department of Internal Medicine, Kobe University Graduate School of Medicine for their technical assistance.

1 Stumvoll, M., Goldstein, B. J. \& van Haeften, T. W. Type 2 diabetes: principles of pathogenesis and therapy. Lancet 365, 1333-1346 (2005).

2 Sladek, R., Rocheleau, G., Rung, J., Dina, C., Shen, L., Serre, D. et al. A genome-wide association study identifies novel risk loci for type 2 diabetes. Nature 445, 881-885 (2007).

3 Scott, L. J., Mohlke, K. L., Bonnycastle, L. L., Willer, C. J, Li, Y, Duren, W. L. et al. A genome-wide association study of type 2 diabetes in Finns detects multiple susceptibility variants. Science 316, 1341-1345 (2007).

4 Dupuis, J., Langenberg, C., Prokopenko, I., Saxena, R., Soranzo, N., Jackson, A. U. et al. New genetic loci implicated in fasting glucose homeostasis and their impact on type 2 diabetes risk. Nat. Genet. 42, 105-U32 (2010).

5 Yasuda, K., Miyake, K., Horikawa, Y., Hara, K., Osawa, H., Furuta, H. et al. Variants in KCNQ1 are associated with susceptibility to type 2 diabetes mellitus. Nat. Genet. 40, 1092-1097 (2008)

6 Unoki, H., Takahashi, A., Kawaguchi, T., Hara, K., Horikoshi, M., Andersen, G. et al. SNPs in KCNQ1 are associated with susceptibility to type 2 diabetes in East Asian and European populations. Nat. Genet. 40, 1098-1102 (2008).

7 Yamauchi, T., Hara, K., Maeda, S., Yasuda, K., Takahashi, A., Horikoshi, M. et al. A genome-wide association study in the Japanese population identifies susceptibility loci for type 2 diabetes at UBE2E2 and C2CD4A-C2CD4B. Nat. Genet. 42, 864-868 (2010).

8 Takeuchi, F., Serizawa, M., Yamamoto, K., Fujisawa, T., Nakashima, E., Ohnaka, K. et al. Confirmation of multiple risk Loci and genetic impacts by a genome-wide association study of type 2 diabetes in the Japanese population. Diabetes 58, 1690-1699 (2009).

9 Mori, S., Tanaka, Y., Takahashi, A., Hirose, H., Kashiwagi, A., Kaku, K et al. Association of CDKAL1, IGF2BP2, CDKN2A/B, HHEX, SLC30A8, and KCNJ11 with susceptibility to type 2 diabetes in a Japanese population. Diabetes 57, 791-795 (2008).

10 Ohshige, T., Iwata, M., Omori, S., Tanaka, Y., Hirose, H., Kaku, K. et al. Association of new loci identified in European genome-wide association studies with susceptibility to type 2 diabetes in the Japanese. PLoS One 6, e26911 (2011).

11 Gibson, G. Rare and common variants: twenty arguments. Nat. Rev. Genet. 13, 135-145 (2011).

12 Alberti, K. G. \& Zimmet, P. Z. Definition, diagnosis and classification of diabetes mellitus and its complications. Part 1: diagnosis and classification of diabetes mellitus provisional report of a WHO consultation. Diabet. Med. 15, 539-553 (1998).

13 Horikoshi, M., Hara, K., Ito, C., Nagai, R., Froguel, P. \& Kadowaki, T. A genetic variation of the transcription factor 7-like 2 gene is associated with risk of type 2 diabetes in the Japanese population. Diabetologia 50, 747-751 (2007).

14 Horikoshi, M., Hara, K., Ito, C., Shojima, N., Nagai, R., Ueki, K. et al. Variations in the HHEX gene are associated with increased risk of type 2 diabetes in the Japanese population. Diabetologia 50, 2461-2466 (2007).

15 Iwata, M., Maeda, S., Kamura, Y., Takano, A., Kato, H., Murakami, S. et al. Genetic risk score constructed using 14 susceptibility alleles for type 2 diabetes is associated with the early onset of diabetes and may predict the future requirement of insulin injections among Japanese individuals. Diabetes Care 35, 1763-1770 (2012).

16 Benjamini, Y. \& Hochberg, Y. Controlling the false discovery rate - a practical and powerful approach to multiple testing. J. Roy. Stat. Soc. Ser. B 57, 289-300 (1995).

$17 \mathrm{Hu}$, C., Zhang, R., Wang, C., Wang, J., Ma, X., Hou, X. et al. Variants from GIPR, TCF7L2, DGKB, MADD, CRY2, GLIS3, PROX1, SLC30A8 and IGF1 are associated with glucose metabolism in the Chinese. PLoS One 5, e15542 (2010).

Supplementary Information accompanies the paper on Journal of Human Genetics website (http://www.nature.com/jhg) 